\title{
Amelioration of bleomycin-induced acute lung injury and epithelial-mesenchymal transition by baicalein in mice
}

\author{
Dheeraj Kumar Sharma ${ }^{1}$, Nittin Dev Singh ${ }^{1 \%}$, Geeta Devi Leishangthem ${ }^{2}$ and \\ Harmanjit Singh Banga ${ }^{1}$ \\ ${ }^{1}$ Department of Veterinary Pathology, and \\ ${ }^{2}$ Animal Disease Research Centre, College of Veterinary Science, Guru Angad Dev Veterinary and Animal Sciences University, \\ Ludhiana 141004 , India
}

During lung fibrosis, acute pulmonary inflammation and epithelial-mesenchymal transition (EMT) play important roles. The present study analyses the ameliorative effect of baicalein, a bioactive flavonoid present in the dry roots of Scutellaria baicalensis Georgi, on bleomycin-induced acute lung injury and subsequent EMT. Mice received a single intratracheal instillation of saline containing bleomycin@1 mg/kg body wt. Baicalein in different doses $(0.1,1.0,10 \mathrm{mg} / \mathrm{kg})$ was given intraperitoneally daily for one week. Pulmonary inflammation and EMT parameters were evaluated. Baicalein significantly attenuated bleomycin-induced pulmonary inflammatory and structural changes. Thus, treatment with baicalein ameliorates bleomycininduced acute inflammation and subsequent early stage of EMT.

Keywords: Acute lung injury, baicalein, bleomycin, epithelial-mesenchymal transition, mice.

ACUTE LUNG INJURY (ALI) includes pulmonary inflammation characterized by alveolar epithelial cell injury, polymorphonuclear cells infiltration along with release of proinflammatory cytokines followed by proliferation of fibroblast and extracellular matrix (ECM) that results in pulmonary fibrosis. The epithelial-mesenchymal transition (EMT) process has been associated with lung fibrosis, where the epithelial cells differentiate and acquire the phenotype of myofibroblasts ${ }^{1}$. In pulmonary fibrosis, resident pulmonary fibroblasts or blood-borne fibrocytes and alveolar epithelial cells (AECs) transdifferentiate to myofibroblasts ${ }^{2,3}$.

Bleomycin (BZM), an antibiotic agent isolated from the fungus Streptomyces verticillus, has been used as an anti-cancer drug, but it causes lung toxicity leading to pulmonary fibrosis as a side effect. Further, bleomycin has been widely used in animals (mice, rats and hamster) to model acute lung injury and pulmonary fibrosis, to

\footnotetext{
*For correspondence. (e-mail: drndsingh@gmail.com)
}

study the mechanism involved in fibrogenesis and to potential therapies ${ }^{4}$. A single dose of bleomycin given intratracheally causes lung injury and fibrosis in rodents ${ }^{5}$. Several studies have reported that bleomycininduced early stages of lung injury resemble human pulmonary fibrosis which includes infiltration of inflammatory cells, higher collagen content and reduced lung volumes and compliance 5 . Further, EMT plays an important roles in the pathogenesis of bleomycin-induced lung injury ${ }^{6}$.

Baicalein (BAIC) is a bioactive flavonoid, present in the dry roots of Scutellaria baicalensis Georgi, which is widely used in traditional Chinese herbal medicine. Evidence has shown that BAIC has many pharmacological effects, including anti-allergic, antioxidant, anti-apoptotic, anti-viral, anti-inflammatory, anti-tumour and a immunemodulatory effects ${ }^{7}$. Importantly, baicalein improves the dysfunction of mitochondria, thereby preventing allergic asthma ${ }^{8}$.

It has been reported that the future therapeutic approach may focus on improvement in regeneration of alveolar epithelial cells ${ }^{9}$ and reversal of the EMT process ${ }^{10}$. Few studies are available on the ameliorative effect of baicalein on pulmonary fibrosis induced by bleomy$\operatorname{cin}^{11,12}$. However, all of them are on 28 days chronic pulmonary fibrosis. The present study analyses the ameliorative effect of baicalein on acute lung injury induced by bleomycin and its subsequent EMT.

\section{Materials and methods}

\section{Animals and treatments}

The study protocol was approved by the Institutional Animal Ethics Committee (IAEC) of Guru Angad Dev Veterinary and Animal Sciences University (GADVASU), Ludhiana, Punjab, India. Male albino mice $(n=30,4-6$ weeks age) were obtained from the Central Research Institute, Kasauli, Himachal Pradesh and maintained in the small animal house at GADVASU. After the 
acclimatization period of seven days, 30 male albino mice were divided randomly into five experimental groups (six animals each) and named according to the challenge and treatment: SHAM/PBS (saline-only/control group), BLM (bleomycin@1 mg/kg treated group), BLM/BAIC/0.1 (bleomycin@1 mg/kg + baicalein $(0.1 \mathrm{mg} / \mathrm{kg}))$, BLM/ BAIC/1 (bleomycin@ $1 \mathrm{mg} / \mathrm{kg}+$ baicalein $(1 \mathrm{mg} / \mathrm{kg})$ ) and BLM/BAIC/10 (bleomycin@ @ $1 \mathrm{mg} / \mathrm{kg}+$ baicalein $(10 \mathrm{mg} / \mathrm{kg}))$. Mice were intratracheally given a single dose of saline containing bleomycin sulphate @ $1 \mathrm{mg} / \mathrm{kg}$ body wt (Sigma, USA) in a volume of $50 \mu 1$ in all groups, except the group vehicle. Baicalein (Sigma, USA) in three different doses@0.1,1.0 and $10 \mathrm{mg} / \mathrm{kg}$ was given intraperitoneally daily for one week. All the mice were sacrificed after 1 week by ketamine and xylazine overdose.

\section{Collection of blood and bronchoalveolar lavage fluid}

After sacrifice, blood was withdrawn by cardiac puncture and collected in EDTA vials for haematological estimation, such as haemoglobin concentration $(\mathrm{Hb})$ and total leucocyte count (TLC). Bronchoalveolar lavage (BAL) was done three times in each animal from the left lung using a tracheal cannula attached to $1 \mathrm{ml}$ syringe with $0.5 \mathrm{ml}$ of PBS. Bronchoalveolar lavage fluid (BALF) was processed to get cell pellets and supernatants as described earlier $^{13}$. Total cell and differential cell counts were done with the cell pellets using a haemocytometer and staining with Leishman stain. BALF supernatants were collected and stored at $-80^{\circ} \mathrm{C}$ for the estimation of protein and cytokines, TNF- $\alpha$ and IL- 6 concentration.

\section{Wet to dry lung weight ratio}

The wet to dry lung weight (W/D) ratio was estimated as an indicator of pulmonary oedema. Similar lobes of the right lung from each sacrificed animal were taken and weighed immediately after excision (wet weight). Then they were desiccated at $60^{\circ} \mathrm{C}$ for three days $(72 \mathrm{~h})$, and weighed again (dry weight). The W/D ratio was calculated for each animal.

\section{Estimation of protein and cytokines}

Protein was estimated in BALF using commercially available BCA Protein Assay Kit (Thermo Scientific, USA), according to the manufacturer's protocol. Interleukin-6 (IL-6) and tumour necrotic factor-alpha (TNF- $\alpha$ ) in BALF and transforming growth factor- $\beta$ in lung homogenates were estimated using Mouse IL-6 and Mouse TNF- $\alpha$ ELISA Kit (Krishgen Biosystem, Mumbai) and Mouse TGF- $\beta$ ELISA Kit (YH Bioresearch Laboratory, Shanghai, China) according to the manufacturer's protocol.

\section{Histopathological studies}

After collection of BALF, lung tissue samples were fixed in $10 \%$ neutral buffered formalin, and processed and embedded in paraffin. Next, $5 \mu \mathrm{m}$ thick sections were stained with routine hematoxylin and eosin, Masson's trichrome and picro-sirius red staining. The slides were observed and photomicrographs taken using a microscope attached with a camera (BX 61, Olympus Corporation, Japan). Lung injury assessment was done by experimentally unknown experts and graded semi-quantitatively using modified Ashcroft's scoring method ${ }^{14}$, with a score range $0-8$ score. For picro-sirius red staining, the slides were stained with picro-sirius red stain $(0.1 \%$ sirius red in aqueous saturated picric acid) for $1 \mathrm{~h}$ followed by washing with acidified water $(0.5 \%$ glacial acetic acid $)$, dehydration and mounting with DPX. Collagen was red in colour, while non-collagen components were orange. The images were analysed using Image J (Fiji) software (http://fiji.sc). The intensity of the picro-sirius red positive area was expressed as percentage area $\left(\mu \mathrm{m}^{2}\right)$.

\section{Estimation of lipid peroxidation and superoxide dismutase}

The lungs (10 mg) were homogenized in $1 \mathrm{ml}$ of ice-cold phosphate buffered saline $(\mathrm{pH}$ 7.4), using a tissue homogenizer with a teflon pestle at $4{ }^{\circ} \mathrm{C}$ and centrifuged to obtain the tissue homogenates which were used for measurement of total protein concentration, lipid peroxidation (LPO) and superoxide dismutase (SOD) activity. Total protein concentration was estimated using commercially available BCA Protein Assay Kit (Thermo Scientific, USA), according to the manufacturer's protocol. LPO was determined in relation to production of MDA (malondialdehyde) using the thiobarbituric acid (TBA) method as described by Shafiq-ur-Rehman ${ }^{15}$, with slight modification. The amount of LPO was expressed in nanomoles of MDA per gram of tissue. SOD activity was assessed according to the method described by Madesh and Balasubramanian ${ }^{16}$.

\section{Estimation of myeloperoxidase activity}

Myeloperoxidase (MPO) activity was measured in lung homogenates using Myeloperoxidase Colorimetric Activity Assay Kit (Sigma-Aldrich, USA), according to the manufacturer's protocol. The optical density (absorbance) was measured at $412 \mathrm{~nm}$ using a microplate reader.

\section{Estimation of collagen by hydroxyproline assay}

Collagen content in the lung homogenates was measured by hydroxyproline (HYP) assay. Briefly, lung homogenates 

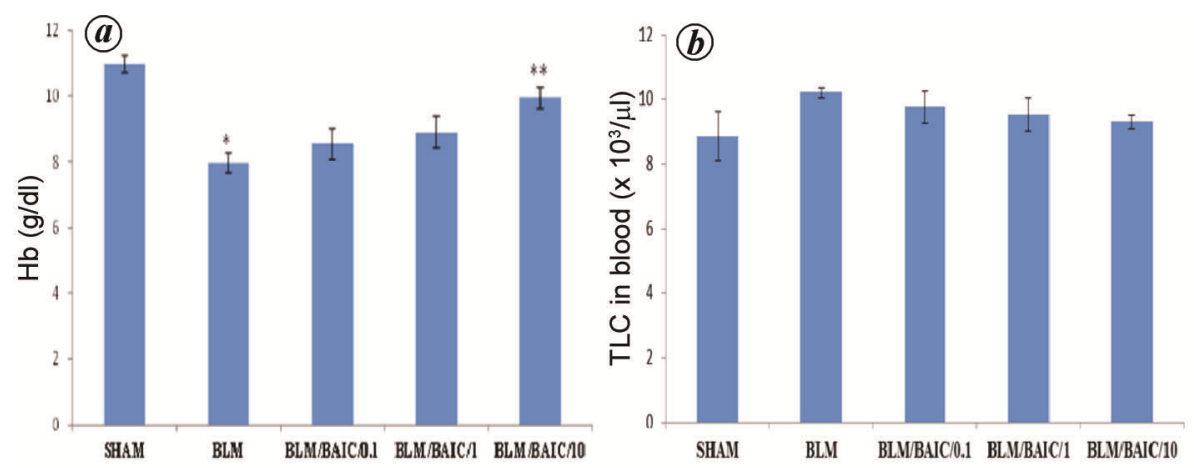

Figure 1. Effect of baicalein on blood hematology in bleomycin-induced lung injury. $\boldsymbol{a}$, Baicalein treatment shows dose-dependent improvement in the level of haemoglobin. $\boldsymbol{b}$, Effect of baicalein on total leukocytes count (TLC) in lung injury induced by bleomycin. Data are mean \pm SE of six mice for each group. ${ }^{*} P<0.05$ versus SHAM group; $* * P<0.05$ versus BLM group.

$(100 \mu \mathrm{l})$ were hydrolysed with equal amount of concentrated $\mathrm{HCl}$ in a pressure-tight, teflon-capped vial at $120^{\circ} \mathrm{C}$ for $3 \mathrm{~h}$, followed by clarification with activated charcoal. The hydrolysed samples $(10 \mu \mathrm{l})$ were transferred to a 96well plate and evaporated to dryness under vacuum. Hydroxyproline standard $(1 \mathrm{mg} / \mathrm{ml})$ was used to prepare the standard curve. To each sample and standard, chloramine $\mathrm{T}$ reagent $(100 \mu \mathrm{l})$ was added and incubated at room temperature for $5 \mathrm{~min}$ followed by $\mathrm{DMAB}$ reagent $(100 \mu \mathrm{l})$ and incubated for $90 \mathrm{~min}$ at $60^{\circ} \mathrm{C}$. Absorbance was read at $560 \mathrm{~nm}$ using a microplate reader.

\section{Immunohistochemistry}

Immunohistochemical (IHC) analysis was performed for epithelial (E-cadherin) and mesenchymal markers (alphasmooth muscle actin $)^{17}$. Briefly, $5 \mu \mathrm{m}$ paraffin sections on poly-L-lysine-coated slides were rehydrated. After heat-induced antigen retrieval in citrate buffer and endogenous peroxidase blocking, the slides were incubated with primary antibodies (mouse monoclonal alphasmooth muscle actin (Abcam, UK) and rabbit polyclonal E-cadherin (Gentex, USA)), followed by incubation with secondary antibody (ABC, Universal, Vector), and colour developed using diaminobenzidine substrate and counterstained with hematoxylin. In the negative control, tissue section was processed without application of primary antibody. Semi-quantitative immunohistochemical analysis was performed using scoring pattern with a score range $0-5$ : 0 (0 positive staining cells $(\%)$, no expression), 1 (less than $1 \%$, negligible expression), 2 (1-10\%, scanty expression), 3 (10-33\%, low-moderate expression), 4 (33-66\%, moderate expression) and $5(>66$, extensive expression) ${ }^{18}$.

\section{Ultrastructural examination}

Lung tissues were dissected at a size of $1 \mathrm{~mm}^{3}$ and fixed in Karnovsky's fixative for $6 \mathrm{~h}$ at $4^{\circ} \mathrm{C}$. The tissues were processed for transmission electron microscopy (TEM) as described earlier ${ }^{19}$. After several washings, lung tissues were post-fixed in $1 \%$ osmium tetraoxide for $1 \mathrm{~h}$ at $4^{\circ} \mathrm{C}$ followed by dehydration at various grades of acetone (30-100\%) for $30 \mathrm{~min}$ each at $4{ }^{\circ} \mathrm{C}$ and clearing with two changes of toluene for $30 \mathrm{~min}$ at room temperature, and further processed and embedded in pure epoxy resin to make blocks. Ultrathin sections $(70 \mathrm{~nm})$ were mounted on a copper grid and stained with uranyl acetate and lead citrate. The tissue sections were visualized with Tecnai $200 \mathrm{kV}$ transmission electron microscope (Tecnai, Fei Electron Optics, Oregon, United States).

\section{Statistical methods}

Data were presented as mean \pm SE. Data of different groups were evaluated using SPSS/10.0 software. To detect differences among groups, one-way analysis of variance (ANOVA) was used and the means were compared by Duncan's and LSD post-hoc test, and a value of $P \leq 0.05$ was taken as significant.

\section{Results}

\section{Effect of baicalein on hematology in bleomycin- induced lung injury}

The mean haemoglobin level in the BLM group (7.96 \pm $0.29 \mathrm{~g} / \mathrm{dl})$ was lower compared to SHAM (10.97 \pm 0.26 $\mathrm{g} / \mathrm{dl})$ and BLM/BAIC/10 group (9.94 $\pm 0.33 \mathrm{~g} / \mathrm{dl}$; Figure $1 a)$. Further, the TLC values were higher in the BLM $\left(10.20 \pm 0.16 \times 10^{3} / \mu \mathrm{l}\right)$ group, which decreased with baicalein treatment $\left(9.75 \pm 0.49 \times 10^{3} / \mu \mathrm{l}\right)$ though it was not significant (Figure $1 b$ ).

\section{Baicalein-attenuated bleomycin-induced pulmonary oedema and microvascular permeability}

The mean W/D lung weight ratio in the BLM group $(8.65 \pm 0.57)$ was significantly $(P<0.05)$ higher compared 

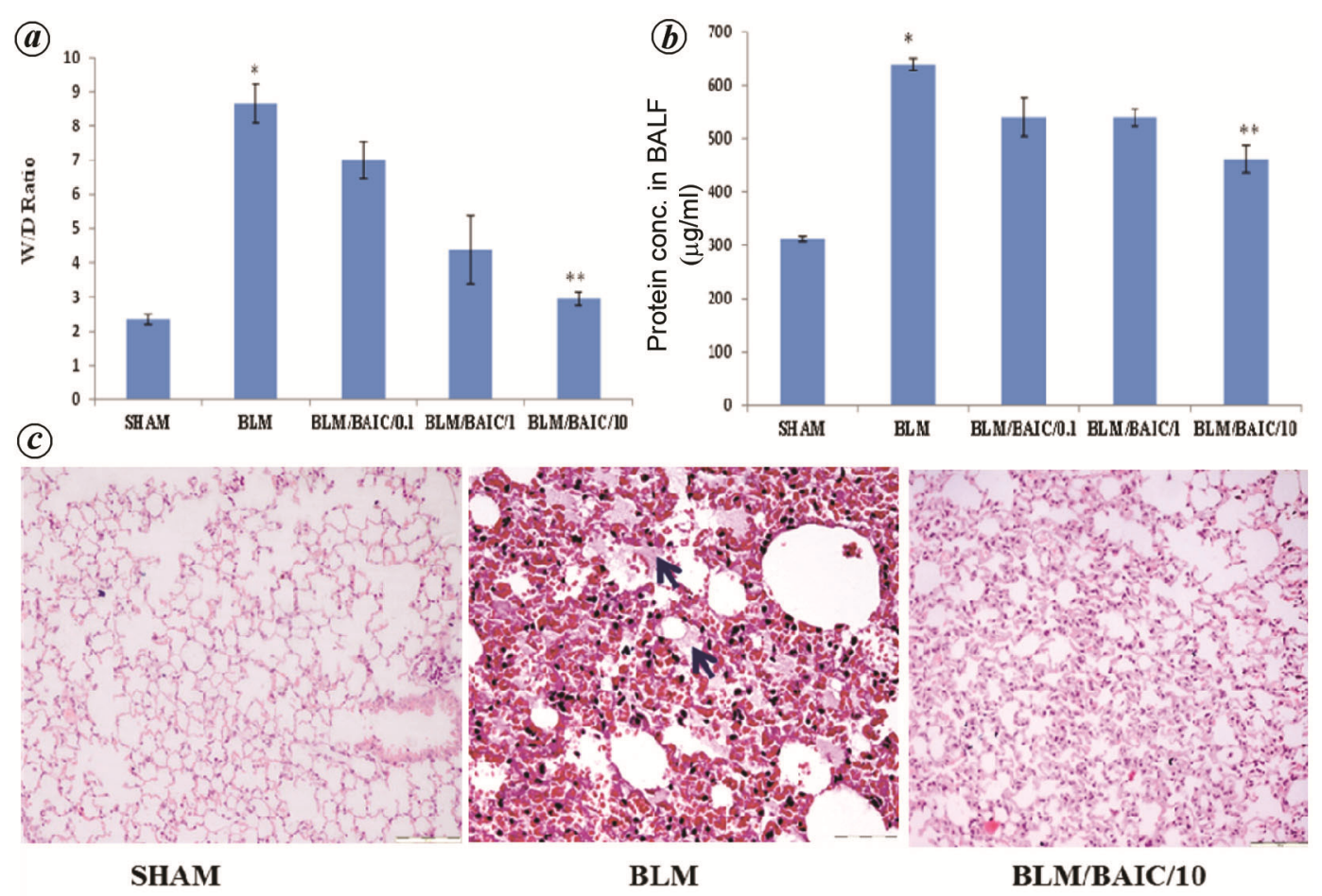

Figure 2. Effect of baicalein on bleomycin-induced pulmonary oedema and microvascular permeability. $\boldsymbol{a}$, The wet dry (W/D) lung weight ratio was calculated in different groups of animals to study pulmonary oedema. $\boldsymbol{b}$, Total protein concentration in BALF estimated as a measure of permeability of blood vessels. $\boldsymbol{c}$, Bleomycin-induced pulmonary oedema as indicated by the presence of pinkish fluid within the alveolar spaces (arrows) attenuated by baicalein treatment (H\&E, bar $=50 \mu \mathrm{m}$ ). Data are mean $\pm \mathrm{SE}$ of six mice for each group. ${ }^{*} P<0.05$ versus SHAM group; ${ }^{* *} P<0.05$ versus BLM group.

to SHAM $(2.34 \pm 0.15)$ and $\mathrm{BLM} / \mathrm{BAIC} / 10 \quad(2.95 \pm$ $0.20)$ groups. The $\mathrm{BLM} / \mathrm{BAIC} / 0.1 \quad(7.00 \pm 0.53)$ and BLM/BAIC/1 (4.39 \pm 1.01$)$ groups showed dose-dependent improvement in W/D lung weight ratio. Moreover, mean W/D lung weight ratio of SHAM and BLM/BAIC/10 groups did not differ significantly, indicating that baicalein (a) $10 \mathrm{mg} / \mathrm{kg}$ attenuated bleomycin-induced lung oedema (Figure $2 a$ ). Further histopathologically, oedema was observed within the pulmonary alveoli in the BLM group which was attenuated with the BLM/BAIC/10 group (Figure $2 b$ ). The total protein concentration in BALF of the BLM group $(638.52 \pm 10.70 \mathrm{mg} / \mathrm{ml})$ was significantly $(P<0.05)$ higher compared to the SHAM $(312.42 \pm$ 4.76). The BLM/BAIC/10 group ( $461.57 \pm 26.72 \mathrm{mg} / \mathrm{ml})$ showed significantly lower total protein concentration compared to the BLM group (Figure 2c).

\section{Baicalein-attenuated bleomycin-induced pulmonary inflammation}

In one week, there was significant increase in TLC in BALF of the BLM groups $\left(2.20 \pm 0.17 \times 10^{3} / \mu \mathrm{l}\right) \mathrm{com}$ pared to the SHAM $\left(0.57 \pm 0.08 \times 10^{3} / \mu 1\right)$ group (Figure $3 a$ ). In contrast, the $\mathrm{BLM} / \mathrm{BAIC} / 10$ group (1.03 \pm $0.06 \times 10^{3} / \mu \mathrm{l}$ ) showed marked reduction in the total num- ber of inflammatory cells. Further, percentage of neutrophils in BALF was lower in the BLM/BAIC/10 group $(62.40 \pm 1.96)$ compared to the BLM group (83.40 \pm 1.31; Figure $3 b$ ). Moreover, mononuclear cells percentage was higher in the BLM/BAIC/10 group (37.60 \pm 1.96) compared to the BLM group (16.60 \pm 1.31 ; Figure $3 c$ ). MPO activity was measured as an indicator of leukocyte (neutrophil) accumulation. Mean MPO activity was significantly higher in the BLM group (225.00 \pm $21.86 \mathrm{mU} / \mathrm{ml})$ compared to the SHAM group $(45.86 \pm$ $2.68 \mathrm{mU} / \mathrm{ml}$; Figure $3 d$ ). However, BLM/BAIC/10 group $(128.01 \pm 4.29)$ showed significant reduction in MPO activity indicating attenuation of the inflammatory cells infiltration, specially neutrophils, due to bleomycin.

When compared to SHAM $(68.67 \pm 2.41 \mathrm{pg} / \mathrm{mg})$, the level of IL-6 was significantly higher in the BLM group $(125.23 \pm 11.01 \mathrm{pg} / \mathrm{mg})$, which was reduced in the $\mathrm{BLM} / \mathrm{BAIC} / 10$ group $(96.24 \pm 2.09 \mathrm{pg} / \mathrm{mg}$; Figure $3 e$ ). Similarly, compared to the SHAM group (49.11 \pm $6.93 \mathrm{pg} / \mathrm{mg}$ ) TNF- $\alpha$ level in the BLM group (177.65 \pm $13.79 \mathrm{pg} / \mathrm{mg}$ ) was higher. However, the BLM/BAIC/10 group $(108.92 \pm 10.87 \mathrm{pg} / \mathrm{mg})$ showed reduced levels of TNF- $\alpha$ (Figure $3 f$ ).

Histopathological studies showed neutrophils and macrophages infiltration in the alveolar areas as well as in perivascular and peribronchiolar areas. There was fibrosis 

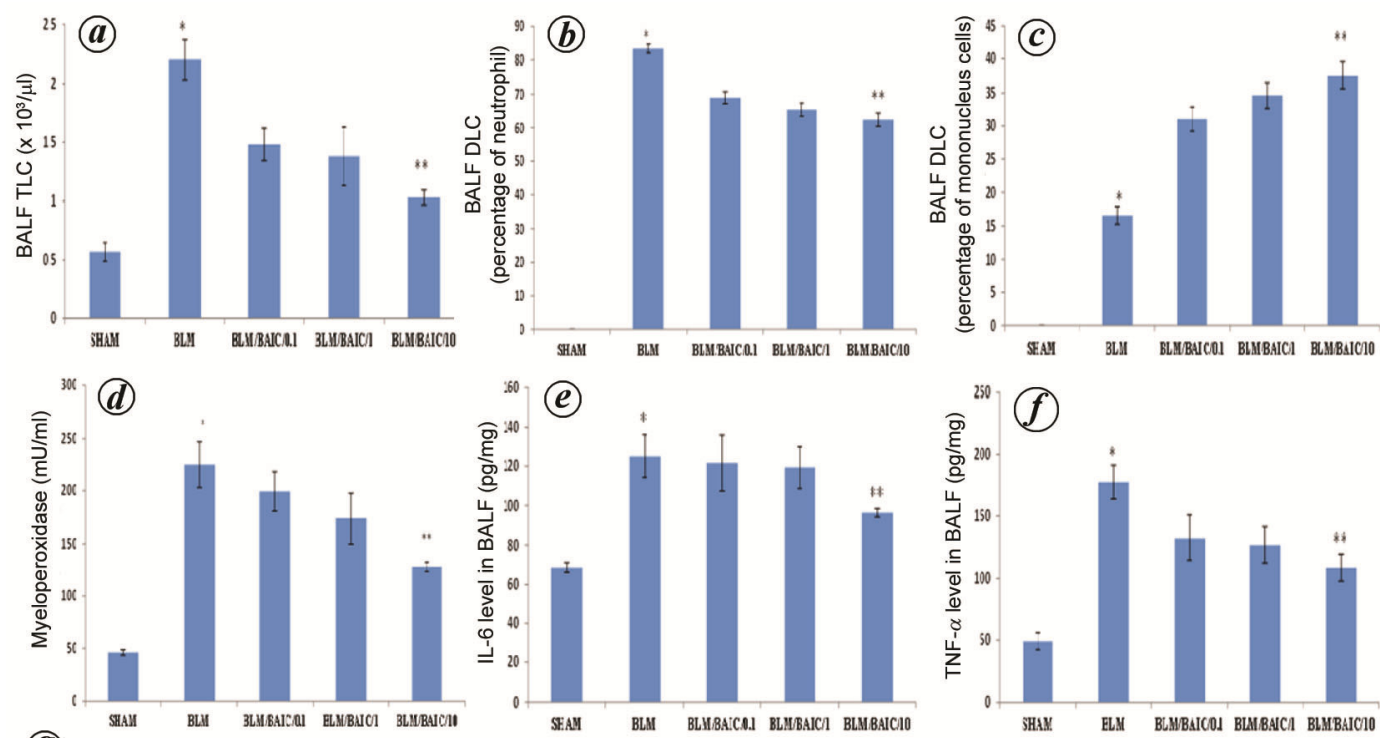

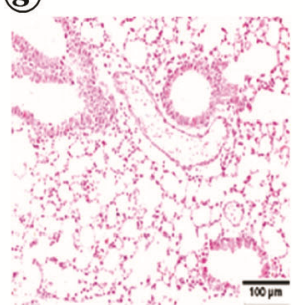

SHAM

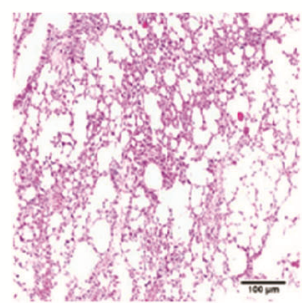

BLM

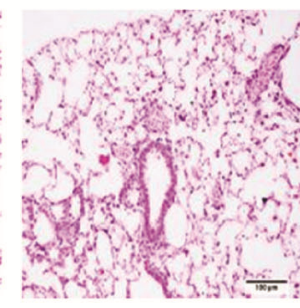

BLM/BAIC/10

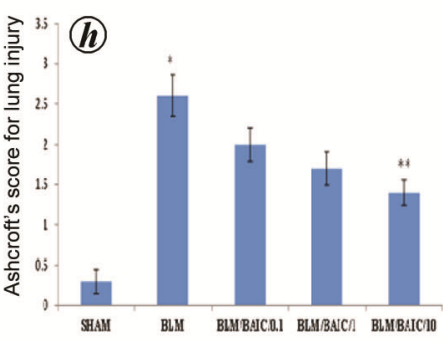

Figure 3. Effect of baicalein on bleomycin-induced pulmonary inflammation. a, Baicalein-attenuated pulmonary inflammatory cells infiltration. TLC in BALF of different groups. $\boldsymbol{b}$, Percentage of neutrophils in BALF increased significantly in BLM groups and reduced in BLM/BAIC/10 group. $c$, Effect of baicalein on mononuclear cells infiltration in bleomycin-induced lung injury. $\boldsymbol{d}$, Myeloperoxidase (MPO) activity measured as an indicator of accumulation of polymorpho nuclear (PMN) leukocyte. Baicalein-attenuated bleomycin-induced accumulation of PMN cells. $\boldsymbol{e}$, Baicaleinattenuated bleomycin-induced pro-inflammatory cytokine (IL-6) production. $f$, Baicalein-attenuated bleomycin-induced inflammatory cytokine (TNF- $\alpha$ ) production. $g$, SHAM group showing normal lung architecture. BLM group showing inflammatory changes and interstitial pulmonary fibrosis. There is infiltration of inflammatory cells within the alveolar areas. BLM/BAIC/10 shows significant alleviation of BLM-induced lung inflammation and fibrosis. (H\&E, bar $=$ $100 \mu \mathrm{m}) . \boldsymbol{h}$, Ashcroft's scoring shows higher lung injury in BLM group, which decreased in BAIC treated groups. Data are mean $\pm \mathrm{SE}$ of six mice for each group. ${ }^{*} P<0.05$ versus SHAM group; $* * P<0.05$ versus BLM group.

in the sub-pleural areas in the BLM-treated group, which was reduced by baicalein treatment. Pulmonary morphological changes as assessed by Ashcroft's scoring scale showed reduction of score in the BAIC-treated group compared to the BLM group (Figure $3 g$ and $h$ ).

\section{Baicalein-attenuated bleomycin-induced oxidative stress}

Figure 4 shows lung MDA and SOD levels in different groups. When compared to the SHAM group (1.61 \pm $0.02 \mathrm{nM} \mathrm{MDA} / \mathrm{g}$ ), lung MDA levels were significantly higher in the BLM group $(2.93 \pm 0.23 \mathrm{nM} / \mathrm{g})$, which decreased in the BLM/BAIC/10 group $(2.12 \pm 0.04 \mathrm{nM}$ MDA/g; Figure $4 a$ ).

SOD activity of the BLM group $(1.84 \pm 0.04 \mathrm{U})$ was significantly lower compared to the SHAM group $(3.85 \pm 0.30 \mathrm{U})$. The BLM/BAIC/0.1 (2.35 $\pm 0.08 \mathrm{U})$,
BLM/BAIC/1 (2.27 $\pm 0.06 \mathrm{U})$ and BLM/BAIC/10 (3.49 \pm $0.03 \mathrm{U})$ groups showed dose-dependent increased in SOD activity compared to the BLM group. SOD activity in the BLM/BAIC/10 group was comparable with the SHAM group (Figure $4 \mathrm{~b}$ ). Thus, baicalein reversed the bleomycin-induced decrease in SOD activity.

\section{Baicalein-attenuated bleomycin-induced pulmonary fibrosis}

To determine the effect of baicalein on early pulmonary fibrosis induced by bleomycin, TGF- $\beta$ level and HYP assays in lung homogenates along with Masson's trichrome and picro-sirius red staining were performed (Figure 5). The TGF- $\beta$ level was higher in the BLM group $(185.89 \pm 11.49)$ compared to the SHAM group $(98.43 \pm 3.76)$. BAIC treatment showed reduction in TGF- $\beta$ level in the lung (Figure $5 a$ ). Further, collagen 

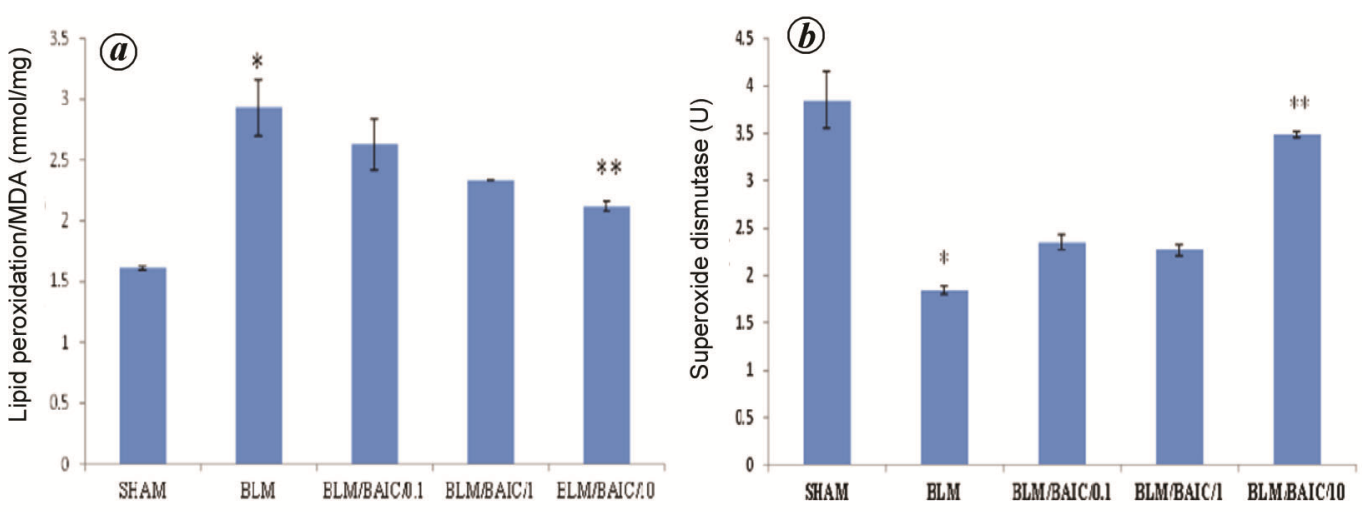

Figure 4. Effect of baicalein on bleomycin-induced oxidative stress. $\boldsymbol{a}$, Baicalein-attenuated bleomycin-induced oxidative stress by lowering the lipid peroxidation (LPO) level, which is increased in BLM groups. $\boldsymbol{b}$, Baicalein-attenuated bleomycin-induced oxidative stress by increasing the superoxide dismutase (SOD) level, which is lower in BLM groups. Data are mean $\pm \mathrm{SE}$ of six mice for each group. ${ }^{*} P<0.05$ versus SHAM group; ${ }^{* *} P<0.05$ versus BLM group.
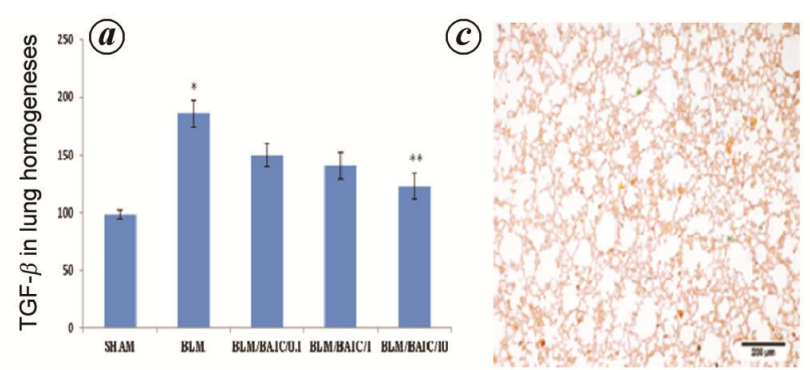

SHAM

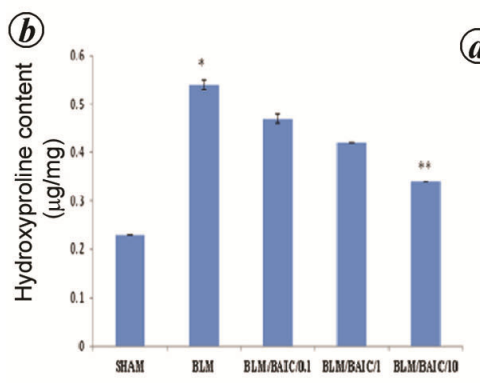

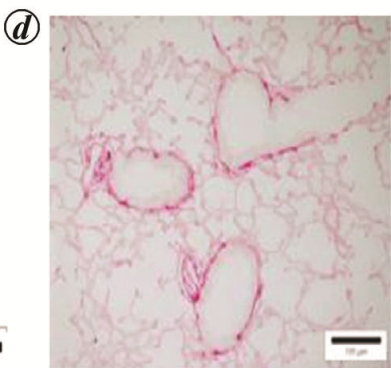

SHAM

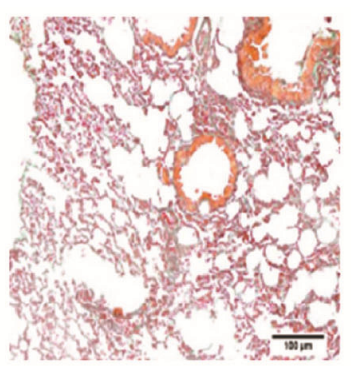

BLM

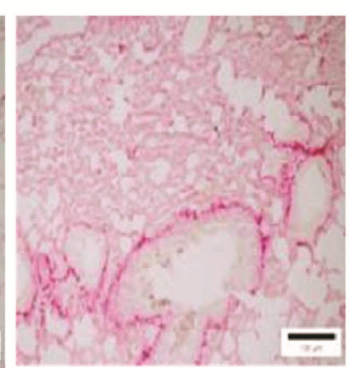

BLM

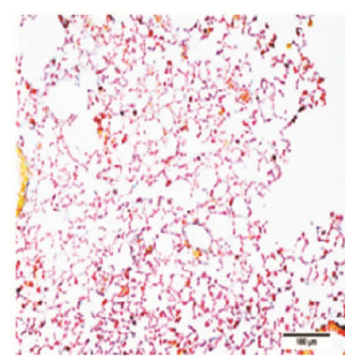

BLM/BAIC/10

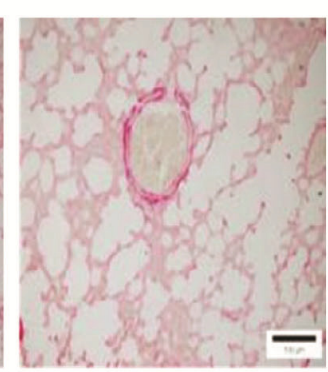

BLM/BAIC/10

Figure 5. Effect of baicalein on bleomycin-induced pulmonary fibrosis. $\boldsymbol{a}$, Baicalein-attenuated bleomycin-induced TGF- $\beta$ production. $\boldsymbol{b}$, Effect of baicalein on bleomycin-induced hydroxyproline/collagen content in different groups. $\boldsymbol{c}$, SHAM mice showing normal histology with normal alveolar architecture. Mild collagen deposition (green colour) is observed in interstitial area in BLM mice. BLM/BAIC/10 shows decreased collagen deposition compared to BLM with improved lung architecture. (Masson's trichrome stain, bar $=200 \mu \mathrm{m}$ (SHAM), $100 \mu \mathrm{m}(\mathrm{BLM}))$. $\boldsymbol{d}$, Normal architecture of lung seen with normal alveoli and normal bronchioles in SHAM mice, while BLM mice, show-decreased alveolar spaces and collagen deposition in interstitial area. BLM/BAIC/10 mice show normal alveoli with mild deposition of collagen in interstitial spaces (picro-sirius red, bar $=100 \mu \mathrm{m}$ ). Data are mean $\pm \mathrm{SE}$ of six mice for each group. $* P<0.05$ versus SHAM group; $* * P<0.05$ versus BLM group.

content examined by HYP assay showed slight significant change in collagen deposition in the BLM group, which was reduced in the BLM/BAIC/10 group $(0.34 \pm 0.00$; Figure $5 b$ ).

Moreover, there was decreased fibrosis in the baicalein-treated groups, as observed in Masson's trichrome and picro-sirius red stained sections. Score of picro-sirius red stained section in \% area was higher in the BLM group $\left(4.23 \pm 1.00 \mu \mathrm{m}^{2}\right)$ compared to the SHAM group $(0.30 \pm$ $\left.0.11 \mu^{2}\right)$. However, this was reduced in the BLM/ BAIC/10 group $\left(1.24 \pm 0.16 \mu \mathrm{m}^{2}\right.$; Figure $5 c$ and $\left.d\right)$.

\section{Baicalein-restored bleomycin-induced changes in the expression of E-cadherin and alpha-smooth muscle actin}

There was reduced expression of epithelial marker Ecadherin in the BLM group compared to the SHAM group (Figure $6 a$ and $b$ ). In the latter group E-cadherin was expressed in the cytoplasm of the bronchial and alveolar epithelial cells with an overall score of $(4.5 \pm$ $0.22)$, whereas the BLM group $(2.33 \pm 0.33)$ showed E-cadherin expression only at the tip of the bronchial 


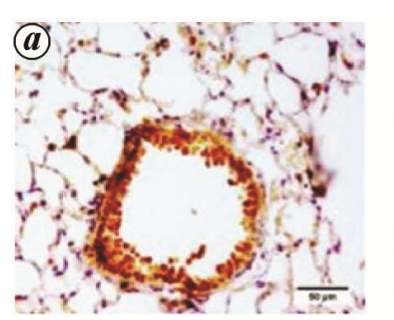

(c)

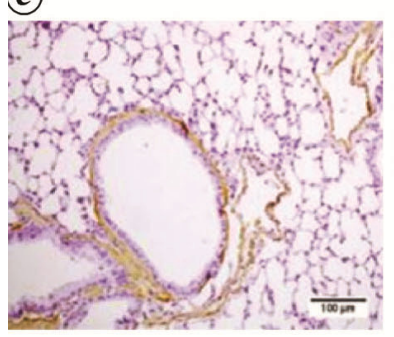

SHAM

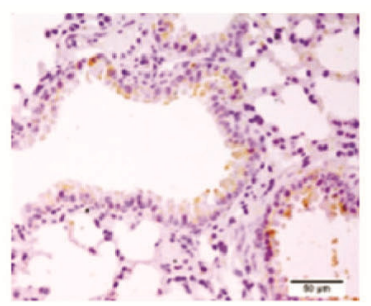

BLM

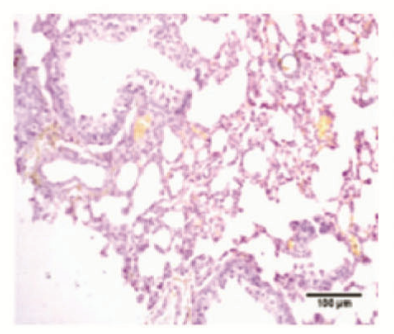

BLM

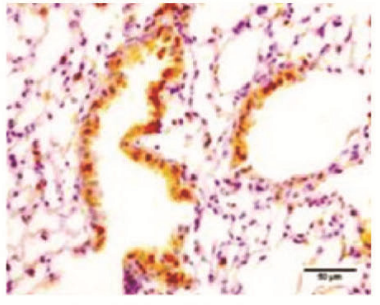

BLM/BAIC/10

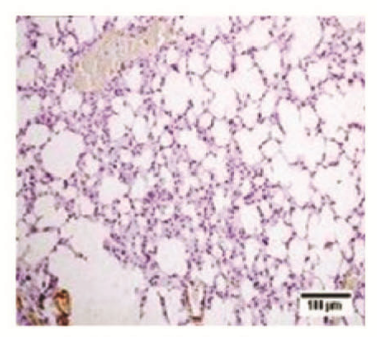

BLM/BAIC/10
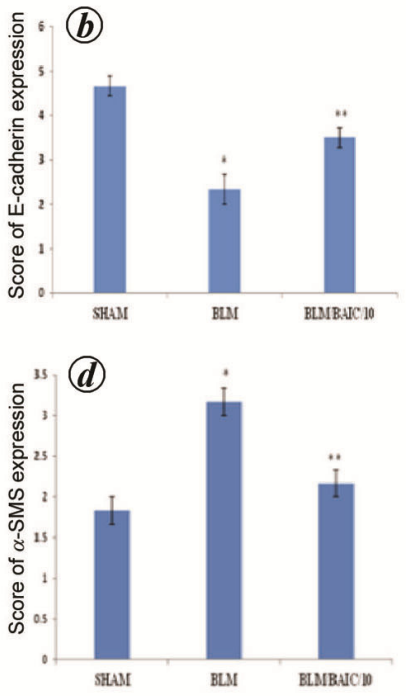

Figure 6. Effect of baicalein on expression of E-cadherin and alpha-smooth muscle actin induced by bleomycin in lungs. $\boldsymbol{a}$, E-cadherin is highly expressed in the cytoplasm of the bronchial epithelial cells and alveolar epithelial cells in SHAM group (brown colour). BLM group shows reduced E-cadherin expression at the tip of the bronchial epithelial cells. BLM/BAIC/10 group restores the expression of E-cadherin in the epithelial cells. (IHC, bar $=50 \mu \mathrm{m}$ ). $\boldsymbol{b}$, Score of E-cadherin expression in lungs of different groups of mice. $\boldsymbol{c}$, Alpha-smooth muscle actin is normally expressed in the bronchial as well as vascular smooth muscles as observed in SHAM. However, $\alpha$-SMA is expressed in the alveolar interstitial areas in BLM mice, which is further reduced by baicalein treatment (IHC, bar $=100 \mu \mathrm{m})$. $\boldsymbol{d}$, Score of alpha-smooth muscle actin expression in the lungs of different groups of mice. Data are mean \pm SE of six mice for each group. $* P<0.05$ versus SHAM group; $* * P<0.05$ versus BLM group.

epithelial cells. However, BAIC treatment restored Ecadherin expression in the epithelial cells $(3.5 \pm 0.22)$. Alpha-smooth muscle actin ( $\alpha$-SMA) was normally expressed in the bronchial as well as vascular smooth muscles as observed in SHAM mice $(1.83 \pm 0.16)$. However, $\alpha$-SMA was expressed in the alveolar interstitial areas in BLM mice $(3.16 \pm 0.16)$, which was further reduced by baicalein treatment $(2.16 \pm 0.16$; Figure $6 c$ and $d$ ).

\section{Baicalein-restored bleomycin-induced ultrastructural changes}

TEM was performed to determine the ultra-morphological changes in bronchial and alveolar epithelial cells. As shown in Figure 7, BLM mice displayed features of cellular injury, where alveolar epithelial cells showed degeneration and apoptotic changes of the nucleus, and swollen mitochondrial cristae. There was aggregation of platelets in the blood vessels of the alveolar areas. Injury of bronchial epithelial cells with loss of mitochondria leading to vacuolation was observed in the BLM group, which was ameliorated in the BLM/BAIC/10 mg group having intact mitochondria with fine cristae. These changes were restored by treatment with baicalein.

\section{Discussion}

In ALI, the main pathological features include early inflammatory injury followed by fibrosis. In the patho- physiology of ALI, inflammation plays an important role; but if uncontrolled leads to excessive tissue injury, chronic inflammation and fibrosis ${ }^{20}$. Single intratracheal injection has been used as a model for both ALI and pulmonary fibrosis since there are two phases of lung injury induced by bleomycin in animal models - acute phase of inflammatory pulmonary injury ${ }^{21}$ and chronic phases of chronic lung injury (pulmonary fibrosis) ${ }^{22}$.

Baicalein is a bioflavonoid with many pharmacological effects $^{7}$ due to its capacity to scavenge the reactive oxygen species (ROS) and improve the status of antioxidants by attenuation of NF- $\kappa$ B activity and suppressing the expression of numerous inflammatory cytokines $^{23}$. Although there are studies on the therapeutic use of BAIC against BLM-induced pulmonary fibrosis, these mainly focus on the later stage (pulmonary fibrosis) occurring at 28 days study $^{11,12}$. Further, the efficacy of BAIC against BLM-induced ALI, especially its effect on EMT status in the initial stage of lung injury is still unknown. In many organs, including the lung, EMT is involved in pathogenesis of fibrosis ${ }^{1}$. Further, in the pathogenesis of bleomycin-induced lung injury, early lungs inflammation and EMT play important roles ${ }^{6,24}$. Acute lung inflammation is always followed by EMT, deposition of collagen and lung fibrosis ${ }^{25}$. Thus, the present study was aimed to examine whether BAIC treatment could ameliorate BLMinduced ALI and the subsequent early stage of EMT in a one-week study. BAIC treatment showed amelioration of BLM-induced ALI and early fibrosis.

A significant decrease in haemoglobin level observed in the BLM group may contribute to the decrease in the 


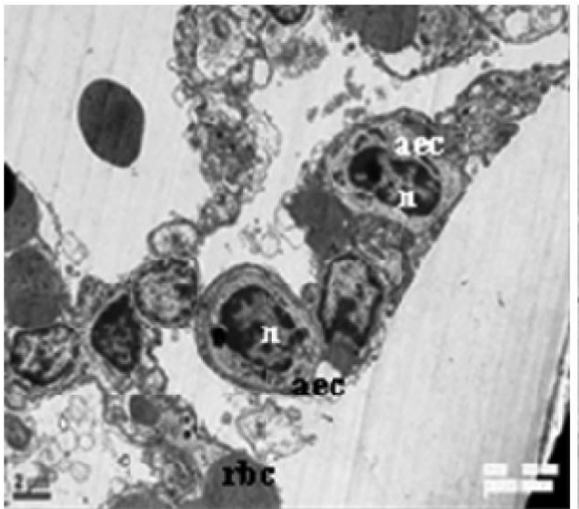

SHAM

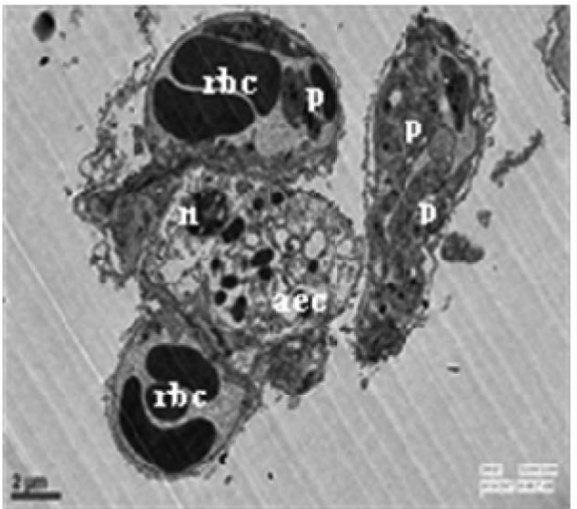

BLM

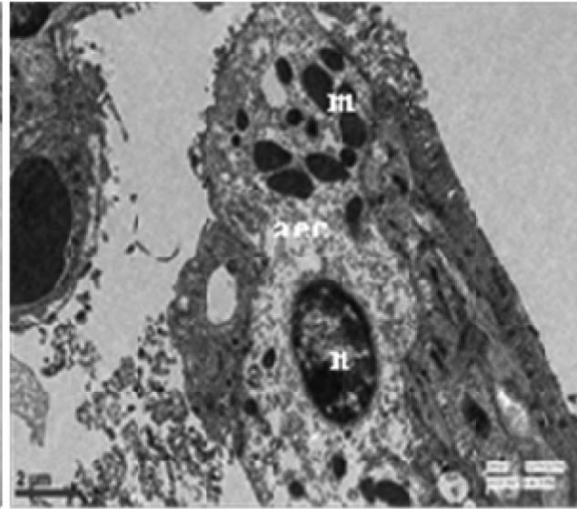

$\mathrm{BLM} / \mathrm{BAIC} / 10$

Figure 7. Representative ultrastructural photomicrographs showing alveolar epithelial cells from different groups of mice. Transmission electron microscopic (TEM) studies show normal nucleus in the alveolar epithelial cells in SHAM. In the BLM group, apoptotic type-2 pneumocyte/alveolar epithelial cells show mitochondrial swelling along with mild mitochondrial and nuclear degeneration. There is also platelet aggregation. $\mathrm{BLM} / \mathrm{BAIC} / 10$ shows restoration of injury to alveolar epithelial cells with improvement in the mitochondrial damage, with fine cristae in the mitochondria ( $n$, nucleus; aec, alveolar epithelial cells; rbc, red blood cell; $\mathrm{p}$, platelet and $\mathrm{m}$, mitochondria; TEM, bar $=2 \mu \mathrm{m}$ ).

level of oxygen as observed mostly in ALI. It has been reported that hypoxia may promote pulmonary inflammatory changes and proliferation of fibroblasts ${ }^{26}$. In the present study, increase in W/D lung weight ratio and protein concentration in BALF of BLM mice might be due to increased lung capillary permeability resulting in an increase in transvascular fluid and protein flux into the interstitium of the lungs ${ }^{27}$. Baicalein treatment not only decreased the W/D lung weight but also decreased the protein concentration in BALF, indicating its protective nature against acute inflammation resulting in pulmonary oedema. BAIC has been reported to markedly attenuate LPS-induced lung oedema in rats ${ }^{28}$.

Further, TLC was higher in BALF and differential cell count showed a major increase in the percentage of neutrophils, while the percentage of mononuclear cells was significantly lower in BLM mice. Infiltration of neutrophils into the lungs is an important constituent of the inflammatory reaction in the initial phase of injury, as neutrophils are the first line of defence ${ }^{29}$. It is recognized that neutrophils accumulation in the lungs is strongly associated with the grave prognosis in septic ALI, and the symptoms of ALI can be prevented is the neutrophil infiltration is inhibited or reduced ${ }^{30}$.

In addition, alveolar inflammatory cells have been considered as a main source of pro-inflammatory cytokines and chemokines release, helping in the accumulation of neutrophils within the tissues, as well as formation of $\mathrm{ROS}^{31}$. Thus, the inflammatory mediators derived from immune cells play an important role in the pathogenesis of $\mathrm{ALI}^{32}$. The present study showed that BAIC significantly reversed the above BLM-induced alterations as depicted by a marked decrease in the number of neutrophils and increase in mononuclear cells in BALF and concomitant decrease in lung MPO activity, a marker of neutrophils accumulation. This might be due to the fact that BAIC inhibited NF- $\kappa$ B activation by augmenting the nuclear factor-erythroid 2-related factor 2/heme oxygenase-1 (Nrf2/HO-1) cascade ${ }^{28}$. Importantly, the increased amount of IL- 6 and TNF- $\alpha$ in BALF of the BLM group was inhibited by BAIC. These findings suggest that the ameliorative effect of BAIC in ALI may be partially accredited to the subdued sequestration and infiltration of inflammatory cells into the lungs, which in turn attenuated the release of pro-inflammatory cytokine.

Bleomycin binds to iron $\left(\mathrm{Fe}^{2+}\right)$ which is involved in redox cycling and catalyses the formation of ROS. During pulmonary fibrosis, ROS target DNA, protein and lipids which ultimately leads to lipid peroxidation ${ }^{33}$. BAIC has an iron or metal chelating activity ${ }^{34}$ and this may be the reason for the decrease in LPO production in the BAIC group as observed in the present study. SOD, a ubiquitous enzyme, protects the cells from detrimental superoxide anion by the process of superoxide dismutation into oxygen and hydroperoxides ${ }^{35}$. A notable decline in SOD activity was observed in the BLM group, which might be due to increased LPO and ROS overproduction ${ }^{36}$. SOD activity was retained to near control animal values upon baicalein treatment. This may be due to the direct action of BAIC on the superoxide radical combined with its capacity to reduce LPO, which in turn decreases production of ROS and oxidative stress during BLM-induced ALI.

Histopathologically, infiltration of PMN in the interstitial area as well the alveolar area, increased alveolar macrophages and deposition of mild fibrin in the interstitial tissues of the BLM group were restored by BAIC that apparently lowered the degree of alveolar inflammation and fibrosis of the lung. Furthermore, anti-fibrotic effect of BAIC in BLM-induced ALI was observed in the 


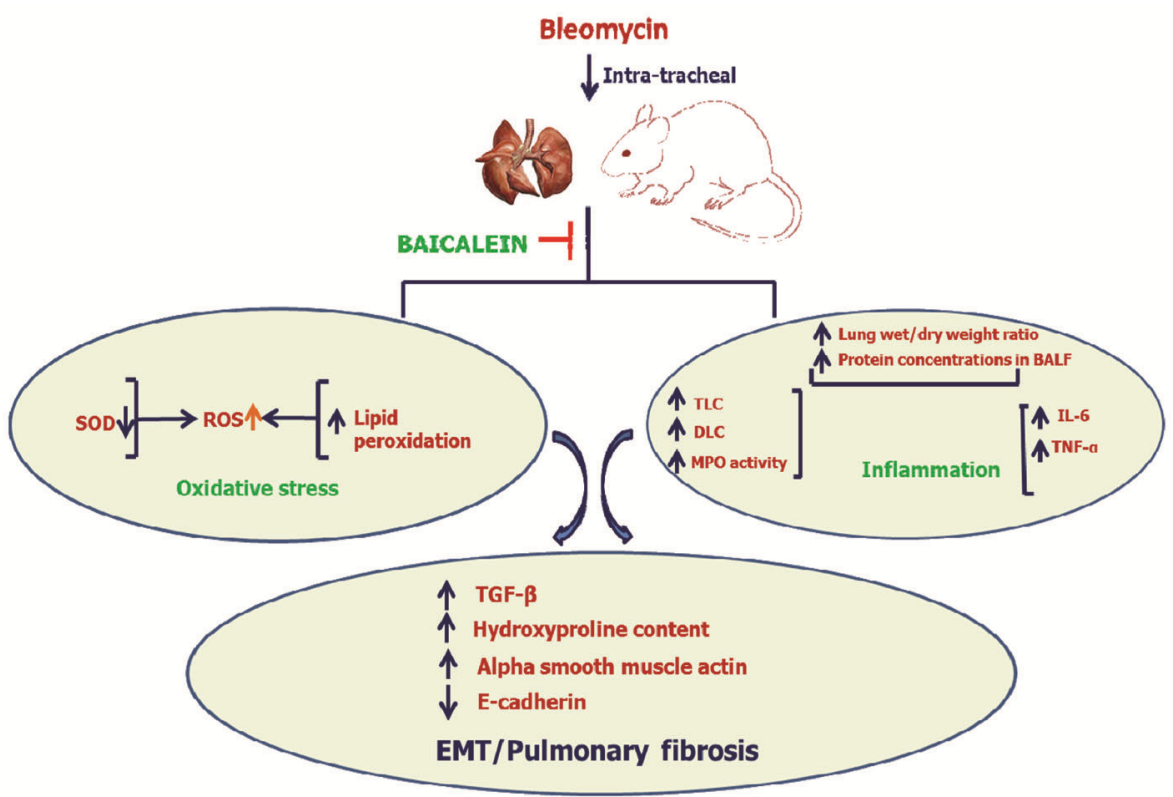

Figure 8. Schematic diagram showing that baicalein ameliorates bleomycin-induced acute lung injury with attenuation of inflammation, oxidative stress and pulmonary fibrosis through reduction of EpithelialMesenchymal Transition.

present study with decrease in the deposition of collagen in the lungs. In ALI, epithelial injury is one of the main pathogenic mechanisms which initiates remodelling of the lungs by inducing various growth factors such as TGF- $\beta$, a potent fibrogenic cytokine. TGF- $\beta$ is increased after bleomycin administration in the epithelial cells, endothelial cells, alveolar macrophages and interstitial fibroblasts, and thereby initiates inflammatory response, apoptosis of epithelial cell and proliferation of fibroblast along with collagen deposition and $\mathrm{EMT}^{37}$. It has been postulated that the initial TGF- $\beta$ activation is due to the initial inflammatory response and generation of $\operatorname{ROS}^{38}$. Baicalein has anti-fibrotic properties in in vitro studies ${ }^{39}$. The decreased collagen deposition in interstitial regions in the BAIC group may be due to inhibition of increased TGF- $\beta 1$ expression and $\mathrm{p}-\mathrm{Smad}-2 / 3$ in bleomycin-treated mice by BAIC ${ }^{12}$.

EMT is a phenomenon where a polarized epithelial cell undergoes multiple biochemical changes and transdifferentiates into a mesenchymal cell phenotype or fibroblast-like cells with increased production of ECM components $^{40,41}$. E-cadherin, a cell-adhesion molecule normally expressed by epithelial cells, is repressed during EMT. During EMT, these epithelial cells leave the epithelial layer and travel through the lining basement membrane followed by accumulation in the tissue interstitium, where they eventually lose the epithelial markers and gain a mesenchymal phenotype ${ }^{42}$. In this study, the expression of E-cadherin was suppressed in BLM mice, which was restored with baicalein treatment. Further, BLM mice showed increased TGF- $\beta$ with decreased expression of E-cadherin. This may be due to the fact that
TGF- $\beta$ represses E-cadherin production in epithelial cells $^{43}$.

Further, $\alpha$-SMA, a contractile protein and actin isoform, which is expressed mainly in the blood vessels of smooth-muscle cells plays an important role in fibrogenesis ${ }^{44}$. In the present study, besides vascular and bronchiolar smooth muscles, $\alpha$-SMA was expressed in the alveolar interstitial areas in BLM mice, which was restored with baicalein treatment. Alpha-SMA-positive myofibroblasts have been demonstrated in type-2 EMT, which is linked with tissue regeneration and organ fibrosis ${ }^{45}$. This occurs due to destabilizing interactions between epithelial cells and/or cell to extracellular membrane ${ }^{46}$. Further, a stressed and injured epithelium can produce myofibroblasts and thereby contribute to fibrogenesis ${ }^{47}$. This indicates that EMT started in the early stage of lung injury induced by bleomycin.

Ultrastructural studies of BLM-treated lungs showed injured and apoptotic alveolar epithelial cells which was restored with baicalein treatment. There was platelet aggregation in the lungs of the BLM group. This may play an important role in the pathogenesis of ALI through recruitment of neutrophils and vascular permeability regulation in the lungs ${ }^{48}$. Baicalein treatment was associated with the restoration of mitochondrial functions with mitochondrial ultrastructural changes in bronchial epithelia, either due to inhibition of 15-LOX or indirectly through reduction of lipid peroxidation ${ }^{8}$. Baicalein may limit apoptosis, probably by prevention of both pathways of apoptosis along with TNF- $\alpha$ production and modulation of pro- and anti-apoptotic signalling elements ${ }^{49}$. 


\section{Conclusion}

In conclusion, baicalein attenuated BLM-induced ALI through suppression of oxidative stress, inflammation, structural damages and EMT, especially during the early stage of injury (Figure 8 ). This study provides additional knowledge on the ameliorative effect of baicalein during the initial stage of lung injury where EMT starts. This may help in the treatment or management of clinical ALIassociated early fibrosis.

1. Chen, C. M., Chou, H. C. and Huang, L. T., Maternal nicotine exposure induces epithelial-mesenchymal transition in rat offspring lungs. Neonatology, 2015, 108, 179-187.

2. Hashimoto, N., Jin, H., Liu, T., Chensue, S. W. and Phan, S. H., Bone marrow-derived progenitor cells in pulmonary fibrosis. J. Clin. Invest., 2004, 113, 243-252.

3. Willis, B. C., Liebler, J. M., Luby, P. K., Nicholson, A. G., Crandall, E. D., du Bois, R. M. and Borok, Z., Induction of epithelialmesenchymal transition in alveolar epithelial cells by transforming growth factor-beta1: potential role in idiopathic pulmonary fibrosis. Am. J. Pathol., 2005, 166, 1321-1332.

4. Moeller, A., Ask, K., Warburton, D., Gauldie, J. and Kolb, M., The bleomycin animal model: a useful tool to investigate treatment options for idiopathic pulmonary fibrosis? Int. J. Biochem. Cell Biol., 2008, 40(3), 362-382.

5. Borzone, G., Moreno, R., Urrea, R., Meneses, M., Oyarzún, M. and Lisboa, C., Bleomycin-induced chronic lung damage does not resemble human idiopathic pulmonary fibrosis. Am. J. Respir. Crit. Care Med., 2001, 63, 1648-1653.

6. Hashimoto, N. et al., Endothelial-mesenchymal transition in bleomycin-induced pulmonary fibrosis. Am. J. Respir. Cell Mol. Biol., 2010, 43, 161-172.

7. Shan, H. et al., The analgesic and anti neuro inflammatory effect of baicalein in cancer-induced bone pain. J. Evid. Based Complement. Altern. Med., 2015; doi:10,1155/2015/973524.

8. Mabalirajan, U. et al., Baicalein reduces airway injury in allergen and IL-13 induced airway inflammation. PLOS ONE, 2013, 8, 62916.

9. Rafii, R., Juarez, M. M., Albertson, T. E. and Chan, A. L., A review of current and novel therapies for idiopathic pulmonary fibrosis. J. Thorac. Dis., 2013, 5, 48-73.

10. Kagalwalla, A. F. et al., Eosinophilic esophagitis: epithelialmesenchymal transition contributes to esophageal remodeling and reverses with treatment. J. Allergy Clin. Immunol., 2012, 129, 1387-1396.

11. Liu, W., Chen, X. L., Liu, J. H., Chen, C. and Ai, J., The effect of baicalein on bleomycin-induced fibrosis in lungs of rats. Chin. J. Appl. Physiol., 2009, 25, 145-149.

12. Gao, Y., Lu, J., Zhang, Y., Chen, Y., Gu, Z. and Jiang, X., Baicalein attenuates bleomycin-induced pulmonary fibrosis in rats through inhibition of miR-21. Pulm. Pharmacol. Ther., 2013, 26, 649-654.

13. Mabalirajan, U., Ahmad, T., Leishangthem, G. D., Joseph, D. A., Dinda, A. K., Agrawal, A. and Ghosh, B., Beneficial effects of high dose of L-arginine on airway hyperresponsiveness and airway inflammation in a murine model of asthma. J. Allergy Clin. Immunol., 2010, 125, 626-635.

14. Hübner, R. H. et al., Standardized quantification of pulmonary fibrosis in histological samples. BioTechniques, 2008, 44, 507517.

15. Shafiq-ur-Rehman, S., Lead-induced regional lipid peroxidation in brain. Toxicol. Lett., 1984, 21, 333-337.
16. Madesh, M. and Balasubramanian, K. A., Microtitre plate assay for superoxide dismutase using MTT reduction by superoxide. Indian J. Biochem. Biophys., 1998, 35, 184-188.

17. Singh, N. D. et al., Effect of feeding graded doses of citrinin on apoptosis and oxidative stress in male Wistar rats through the F1 generation. Toxicol. Ind. Health, 2013, 32, 385-397.

18. Lomas, N. J., Watts, K. L., Akram, K. M., Forsyth, N. R. and Spiteri, M. A., Idiopathic pulmonary fibrosis: immunohistochemical analysis provides fresh insights into lung tissue remodelling with implications for novel prognostic markers. Int. J. Clin. Exp. Pathol., 2012, 5(1), 58-71.

19. Leishangthem, G. D., Mabalirajan, U., Singh, V. P., Agrawal, A., Ghosh, B. and Dinda, A. K., Ultrastructural changes of airway in murine models of allergy and diet-induced metabolic syndrome. ISRN Allergy, 2013; doi:10,1155/2013/261297.

20. Serhan, C. N. et al., Reduced inflammation and tissue damage in transgenic rabbits overexpressing 15-lipoxygenase and endogenous anti-inflammatory lipid mediators. J. Immunol., 2003, 171, 6856-6865.

21. Goto, H., Ledford, J. G., Mukherjee, S., Noble, P. W., Williams, K. L. and Wright, J. R., The role of surfactant protein A in bleomycin-induced acute lung injury. Am. J. Respir. Crit. Care Med., 2010, 181, 1336-1344.

22. Mutsaers, S. E., Foster, M. L., Chambers, R. C., Laurent, G. J. and McAnulty R. J., Increased endothelin-1 and its localization during the development of bleomycin-induced pulmonary fibrosis in rats. Am. J. Respir. Cell. Mol. Biol., 1998, 18, 611-619.

23. Dinda, B., Dinda, S., DasSharma, S., Banik, R., Chakraborty, A. and Dinda, M., Therapeutic potentials of baicalin and it's aglycone, baicalein against inflammatory disorders. Eur. J. Med. Chem., 2017, 131, 68-80.

24. Tanjore, H. et al., Contribution of epithelial-derived fibroblasts to bleomycin-induced lung fibrosis, Am. J. Respir. Crit. Care Med., 2009, 180, 657-665.

25. Li, L. F. et al., Mechanical ventilation augments bleomycininduced epithelial-mesenchymal transition through the Src pathway. Lab. Invest., 2014, 94(9), 1017-1029.

26. Geng, X., Dufu, K. and Hutchaleelaha, A., Increased hemoglobinoxygen affinity ameliorates bleomycin-induced hypoxemia and pulmonary fibrosis. Physiol. Rep., 2006; doi:10,14814/phy2, 12965.

27. Ware, L. B. and Matthay, M. A., Clinical practice, acute pulmonary edema. N. Engl. J. Med., 2005, 353, 2788-2796.

28. Tsai, C. L., Lin, Y. C., Wang, H. M. and Chou, T. C., Baicalein, an active component of Scutellaria baicalensis, protects against lipopolysaccharide-induced acute lung injury in rats. J. Ethnopharmacol., 2014, 153, 197-206.

29. Reutershan, J., Basit, A., Galkina, E. V. and Ley, K., Sequential recruitment of neutrophils into lung and bronchoalveolar lavage fluid in LPS-induced acute lung injury. Am. J. Physiol.-Lung Cell. Mol. Physiol., 2005, 289, 807-815.

30. Grommes, J. and Soehnlein, O., Contribution of neutrophils to acute lung injury. Mol. Med., 2011, 17, 293-307.

31. Agouridakis, P., Kyriakou, D., Alexandrakis, M. G., Prekates, A., Perisinakis, K., Karkavitsa, N. and Bouros, D., The predictive role of serum and bronchoalveolar lavage cytokines and adhesion molecules for acute respiratory distress syndrome development and outcome. Respir. Res., 2002, 3, 25.

32. Bhatia, M. and Moochhala, S. J., Role of inflammatory mediators in the pathophysiology of acute respiratory distress syndrome. $J$. Pathol., 2004, 202, 145-156.

33. Martin, W. J. and Kachel, D. L., Bleomycin-induced pulmonary endothelial cell injury: evidence for the role of iron-catalyzed toxic oxygen-derived species. J. Lab. Clin. Med., 1987, 110, 153-158.

34. Yoshino, M. and Murakami, K., Interaction of iron with polyphenolic compounds: application to antioxidant characterization. Anal. Biochem., 1998, 257, 40-44. 


\section{RESEARCH ARTICLES}

35. McCrod, J. M. and Keele, B. B., An enzyme-based theory of obligate anaerobiosis: the physiological function of superoxide dismutase. Proc. Natl. Acad. Sci. USA, 1971, 68, 1024-1027.

36. Iraz, M. et al., Ginkgo biloba inhibits bleomycin-induced lung fibrosis in rats. Pharmacol. Res., 2006, 53, 310-406.

37. Andre, P. A. et al., BARD1 mediates TGF- $\beta$ signaling in pulmonary fibrosis. Respir. Res., 2015, 29, 118.

38. Barcellos-Hoff, M. H. and Dix, T. A., Redox-mediated activation of latent transforming growth factor-beta 1. Mol. Endocrinol., 1996, 10, 1077-1083.

39. $\mathrm{Hu}, \mathrm{Q}$. et al., In-vitro anti-fibrotic activities of herbal compounds and herbs. Nephrol. Dial. Transplant., 2009, 24, 3033-3041.

40. Lee, J. M., Dedhar, S., Kalluri, R. and Thompson, E. W., The epithelial-mesenchymal transition: new insights in signaling, development, and disease. J. Cell Biol., 2006, 172, 973-981.

41. Schneider, M., Hansen, J. L. and Sheikh, S. P., S100A4: a common mediator of epithelial-mesenchymal transition, fibrosis and regeneration in diseases. J. Mol. Med. (Berl), 2008, 86, 507-522.

42. Kalluri, R. and Weinberg, R. A., The basics of epithelialmesenchymal transition. J. Clin. Invest., 2009, 119, 1420-1428.

43. Choi, J., Park, S. Y. and Joo, C. K., Transforming growth factorbeta1 represses E-cadherin production via slug expression in lens epithelial cells. Invest. Ophthalmol. Vis. Sci., 2007, 48, 2708 2718.

44. Kawasaki, Y. et al., Renal expression of alpha-smooth muscle actin and c-met in children with Henoch-Schonlein purpura nephritis. Pediatr. Nephrol., 2008, 23, 913-919.

45. Zeisberg, M., Hanai, J., Sugimoto, H., Mammoto, T., Charytan, D., Strutz, F. and Kalluri, R., BMP-7 counteracts TGF-beta1- induced epithelial-to-mesenchymal transition and reverses chronic renal injury. Nature Med., 2003, 9, 964-968.

46. Boyer, B., Valles, A. M. and Edme, N., Induction and regulation of epithelial mesenchymal transitions. Biochem. Pharmacol., 2000, 60, 1091-1099.

47. Lee, K. and Nelson, C. M., New insights into the regulation of epithelial-mesenchymal transition and tissue fibrosis. Int. Rev. Cell Mol. Biol., 2012, 294, 171-221.

48. Zarbock, A. and Ley, K., The role of platelets in acute lung injury (ALI). Front. Biosci . J. Virtual Lib., 2009, 14, 150-158.

49. Lai, C. C., Huang, P. H., Yang, A. H., Chiang, S. C., Tang, C. Y., Tseng, K. W. and Huang, C. H., Baicalein attenuates lung injury induced by myocardial ischemia and reperfusion. Am. J. Chin. Med., 2017, 45, 791-811.

ACKNOWLEDGEMENTS. We thank the Science and Engineering Research Board, Department of Science and Technology, Government of India for providing financial support through a project (No. YSS/2014/000045). We also thank Dr Nitika Thakur and Dr Gulzar Begum (Guru Anand Dev Veterinary and Animal Sciences University, Ludhiana) for help, and the Sophisticated Analytical Instrument Facility, All India Institute of Medical Sciences, New Delhi for the use of transmission electron microscopy.

Received 31 October 2018; revised accepted 16 April 2019

doi: $10.18520 / \mathrm{cs} / \mathrm{v} 119 / \mathrm{i} 6 / 962-972$ 